\title{
Miotomía esofágica endoscópica peroral para el tratamiento de la acalasia
}

\author{
Alejandro Nieponice* y Fabio Nachman
}

Fundación Favaloro, Instituto del Esófago, Hospital Universitario, Ciudad Autónoma de Buenos Aires, Argentina

\section{RESUMEN}

La práctica de la miotomía endoscópica peroral (POEM) para el tratamiento de la acalasia ha crecido vertiginosamente en los últimos cinco años y se está transformando en la primera opción terapéutica en varios centros especializados del mundo. La presente revisión detalla los orígenes de esta opción terapéutica, sus indicaciones, la técnica, un análisis de los resultados internacionales y una reseña de la experiencia de nuestro grupo. El trabajo analiza también el reflujo posmiotomía y su comparación con la miotomía laparoscópica de Heller, que es el gran punto de debate en estos tiempos. Los resultados actuales son prometedores, demuestran su seguridad y una recuperación más rápida del paciente, lo cual apoya la adopción del POEM como primera línea de tratamiento de la acalasia en un futuro. Se requiere sin embargo contar con un equipo multidisciplinario con amplia experiencia en endoscopia terapéutica, por lo cual es aconsejable limitar este procedimiento a centros de referencia y con alto volumen en esta patología. En conclusión, la seguridad y eficacia del POEM apoyan un cambio de paradigma respecto a la miotomía laparoscópica para el tratamiento de la acalasia. (NeuroGastroLatam Rev. 2017;1:156-162) Corresponding author: Alejandro Nieponice, anieponi@ffavaloro.org

Palabras clave: POEM. Acalasia. Miotomía endoscópica. Trastorno motor. 


\section{ABSTRACT}

Endoscopic myotomy for the treatment of achalasia (per-oral endoscopic myotomy [POEM]) has grown rapidly in the last 5 years and is becoming the first therapeutic option in several specialized centers worldwide. The present summary details the origins of the technique, its indications, the technical aspects of the procedure, an analysis of the international results and a summary of the results of our group. The work also analyzes the post-myotomy reflux and its comparison with the laparoscopic Heller myotomy, which is the great point of debate in these times. Current results are promising and allow to think in POEM as a first line therapy for achalasia in a near future.

Key words: POEM. Achalasia. Endoscopic myotomy. Motor disorder.

\section{INTRODUCCIÓN}

La acalasia es un trastorno de la motilidad esofágica poco frecuente. Se caracteriza por la pérdida de peristalsis del cuerpo esofágico y la ausencia o falta de adecuada relajación del esfínter esofágico inferior (EEI), debido a una alteración selectiva de las neuronas inhibidoras a dicho nivel. Actualmente no hay un tratamiento curativo que revierta la fisiopatología de la enfermedad. Las alternativas se dirigen a mejorar el paso del bolo alimenticio a través de la unión gastroesofágica (UGE) e incluyen la miotomía quirúrgica y métodos endoscópicos como la dilatación con balón y la inyección de toxina botulínica orientados a debilitar o relajar el EEI ${ }^{1}$.

En los últimos años, la aparición de una nueva alternativa de tratamiento endoscópico ha ganado adeptos a lo largo de todo el mundo debido a la baja morbilidad y a los resultados alentadores en el diferentes series. Esta modalidad es la POEM².

La presente revisión tiene como objetivos describir el POEM, sus indicaciones, así como la experiencia existente, incluyendo las complicaciones con dicha técnica publicadas en la literatura médica.

\section{ANTECEDENTES DEL POEM}

El desarrollo de la cirugía a través de orifítcios naturales a principio del nuevo milenio comportó una gran inversión en desarrolio e investigación de instrumental que mass tarde facilitaría el intervencionismo endoscópico del esófago. Así, con el fin de permi̊ tir resecciones mucosas en bloque o salir a través de la pared del tubo digestivo mè diante la confección de un túnel submucoso se desarrollaron técnicas en donde un alērón mucoso servía de protección sellante entre el orificio de entrada al túnel y el de salida hacia la cavidad mediastinal o abdỡ minal. Los estudios en animales confirma ron la viabilidad y la seguridad de salir dē esófago hacia el mediastino a través un miotomía de entre 2 y $5 \mathrm{~cm}$. Un estudio en animales posterior confirmó que la endoscopia submucosa combinada con la miotomía de la capa circular interna podía reducir 
la presión en el EEI en cerdos ${ }^{3}$. Poco después, adaptando el principio de la técnica endoscópica de la disección submucosa, se realizó el primer POEM ${ }^{2,4-6}$.

\section{INDICACIONES Y CONTRAINDICACIONES}

Los informes iniciales de las diferentes series publicadas presentan el POEM como una alternativa eficaz y de baja morbilidad para el tratamiento de la acalasia clásica ${ }^{4}$.

Existen también buenos resultados reportados en casos de otros trastornos motores, como el espasmo esofágico difuso, el EEI hipertensivo, el esófago en cascanueces y el esófago en Jackhammer. En este grupo particular de pacientes, el resultado de la miotomía laparoscópica no es tan efectivo en cuanto al tratamiento del dolor como lo es con la disfagia. Probablemente la disminución de la eficacia en estos casos se debe a que la alteración motora es más extensa. Con base en lo expuesto y en la opinión de expertos en el tema se recomienda el POEM en estos trastornos motores difusos y no la miotomía de Heller clásica, ya que la misma se puede realizar en mayor extensión desde una zona más proximal en el esófa$\mathrm{go}^{7}$.

\section{EL POEM LUEGO DE FRACASO DE LOS TRATAMIENTO CLÁSICOS}

Existen varios escenarios posibles, con mayor o menor posibilidad de éxito. En los casos de inyecciones con toxina botulínica y las dilataciones endoscópicas existen diferentes grados de fibrosis de la capa submucosa, que conllevan una mayor dificultad a la hora de encontrar los planos de disección ${ }^{8}$. No obstante, la opinión de la mayoría de los expertos es que, si bien los tratamientos previos dificultan el procedimiento, puède llevarse a cabo en grupos experimenta dos. En pacientes que han sido sometidos una miotomía de Heller, una nueva miotê. mía en la cara anterior expone a un riesgo elevado de apertura mucosa con las posibles complicaciones que ello comporta; contră riamente, un POEM realizada en hora (cara posterior) elimina esta posibilidad ầ transcurrir por tejido virgen y añade la pösibilidad de realizar una mejor miotomía Quizás esta es la indicación más clara y rèlevante para este método.

\section{LA TÉCNICA DEL POEM}

Para poder realizar una miotomía endoscóopica se precisa disponer de modo generà de un sobretubo de trabajo, ya que el pröcedimiento puede demandar la entrada y salida del equipo varias veces; un videoendoscopio de alta resolución, idealmente tềrapéutico (se puede utilizar uno con canạl simple de diagnóstico) y un insuflador de de $\mathrm{CO}_{2}$. Como materiales de trabajo intralum nal se requiere de un capuchón semirrígidō, un inyector, una pinza de hemostasia de agarre y un cuchillo endoscópico con una fuente de energía monopolar con modo spray $^{2}$.

Para iniciar el procedimiento se realiza una videoendoscopia digestiva alta (VEDA), mediante la cual se tomarán las medidas correspondientes a la UGE, el hiato y el 
sitio donde se realizará la mucosectomía. Del mismo modo, esta primera VEDA servirá de parámetro para evaluar el pasaje y resalto a nivel de la UGE. El sitio de la mucosectomía se ubica por lo menos $15 \mathrm{~cm}$ distante de la UGE e idealmente en hora 2 de la circunferencia. Se realiza un habón en el sitio seleccionado inyectando solución fisiológica teñida con índigo carmín para generar un buen espacio submucoso. Se realiza una mucosectomía con el cuchillo de aproximadamente $15 \mathrm{~mm}$, lo que permite el ingreso cómodo del endoscopio. Bajo visión directa, se inicia la confección de un túnel submucoso hasta pasar la UGE al menos $3 \mathrm{~cm}$ distal $^{2}$. Para facilitar esta maniobra se realizan sucesivas infiltraciones con la solución antes mencionada, a fin de proteger la mucosa del daño térmico y ampliar el campo de trabajo. Durante la tunelización pueden aparecer vasos sanguíneos que deberán ser coagulados utilizando la pinza de hemostasia de agarre. Completado el túnel submucoso se inicia la miotomía propiamente dicha en dirección cefalocaudal, dejando una superposición mucosomuscular de al menos $5 \mathrm{~cm}$ en la porción proximal. Este aleron servirá de válvula de seguridad en caso de apertura de todo el espesor de la capa muscular. Si bien la indicación precisa de la miotomía es solo de la capa circular, ya hay trabajos publicados que hablan de la seguridad de la sección de toda la capa muscular ${ }^{9}$. Completada la miotomía se instilará una solución de antibióticos para flora entérica en el túnel y se completará el procedimiento con el cierre de la mucosectomía mediante la colocación de clips, habitualmente en número de 4 (Fig. 1).

\section{RESULTADOS}

El trabajo publicado por el grupo Natural Orifice Surgery Consortium for Assessment and Research (NOSCAR) sobre el POEM proporcionó una amplia revisión y tabulación de resultadồs de eficacia con trabajos publicados hasta priầcipios de $2014^{10}$. Estas primeras series en genè ral tuvieron una mediana de seguimiento de un año o menos, y muchas de ellas tenían un muy pequeñonúmerode pacientes (de15a 30) $)^{2}: \frac{11}{6}$. La eficacia fue medida mediante el parámetro utilizado por la mayoría de los estudios reali zados en las últimas dos décadas, una dismínución de la puntuación de Eckardt (ES) a $3 \%$ menos. La ES evalúa el conjunto de síntomăs relacionados a la acalasia, incluyendo dolor, disfagia, pérdida de peso y regurgitaciones; se valora de 0 a 12 y se considera que una puño tuación de menos de 3 significa problema

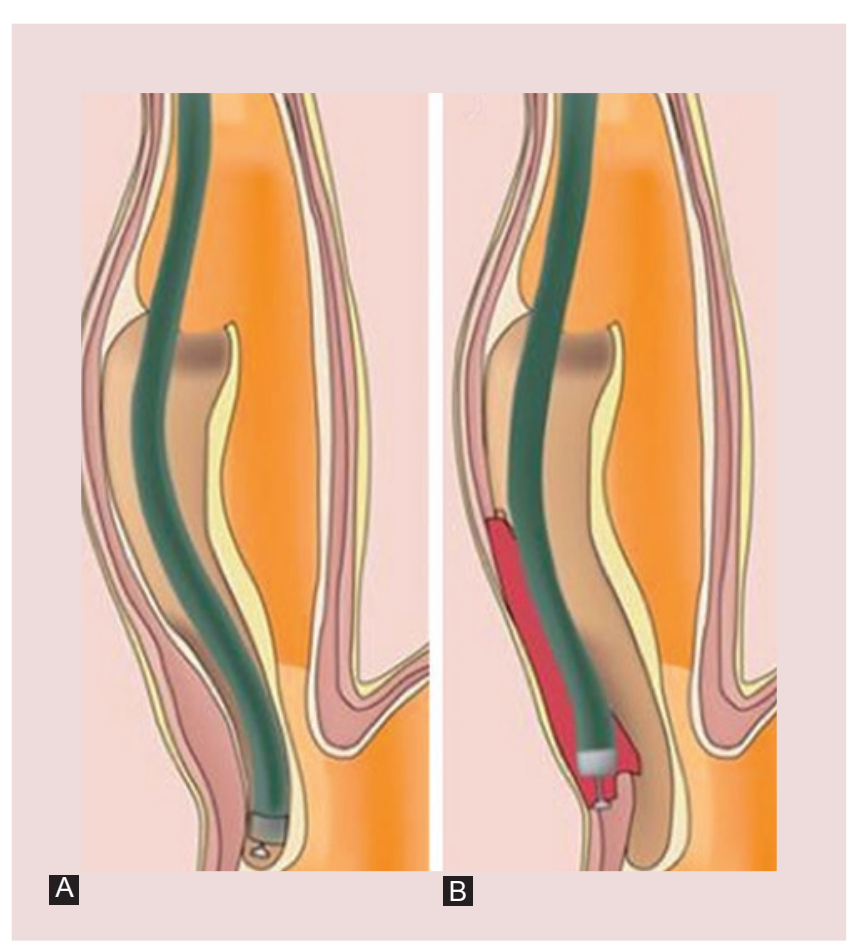

FIGURA 1. Esquema del procedimiento. A: mucosectomía y creación del túnel; B: miotomía. 
resuelto $^{12}$. La eficacia fue uniformemente excelente $(90-100 \%$ a los 3-12 meses), excepto en la serie del estudio multicéntrico europeo, donde fue del $82 \%$ en los pacientes que completaron un año de seguimiento ${ }^{13}$.

Los estudios han demostrado que los centros necesitan realizar un mínimo de 20 a 40 procedimientos para completar la curva de aprendizaje $^{14,15}$. En los últimos dos años, nuevas publicaciones han aportado datos con seguimiento a más largo plazo. Cuatro series occidentales de los centros pioneros en Portland (EE.UU.), Chicago (EE.UU.), Minnesota (EE.UU.) y Roma (Italia), con 100, 41, 93 y 100 pacientes respectivamente, informaron tasas de éxito clínico del $82 \%, 92 \%, 93 \%, 96 \%$ y $94 \%$ en un seguimiento medio de 21, 5, 12, 22, y 11 meses, respectivamente (Tabla 1) $)^{15,16}$. En la mayor serie de casos hasta la fecha, Inoue, et al., informaron los resultados en 500 pacientes, con 105 pacientes con más de tres años de seguimiento ${ }^{17}$. El procedimiento se realizó con éxito en todos los pacientes. Eventos adversos moderados ocurrieron en un 3.2\% eincluyeron neumotórax, sangrado, lesiones de la mucosa, hematomas posoperatorios, derrame pleural y la inflamación del epiplón menor. La mayoría fueron tratados de forma conservadora. No hubo eventos adversos graves. El éxito clínico se logró en el 91.7\%. En el seguimiento endoscópico, el 65\% tenían signos de esofagitis por reflujo, pero solo el $17 \%$ de los pacientes reportó síntomas de enfermedad por reflujo gastroesofágico (ERGE $)^{17}$. A los tres años, el éxito global se mantuvo alto, en èl $88.5 \%$, con ERGE sintomática en el 21\% y signồ de esofagitis por reflujo en el $56 \%$ de los caso Todos los síntomas de reflujo se controlaron eficazmente con inhibidores de la bomba de prồtones (IBP) $)^{17}$.

\section{EL POEM Y LA ENFERMEDAD POR REFLUJO GASTROESOFÁGICO}

El problema de la ERGE después del POEM ès de gran interés, porque está desplazando rápî̀damente a la miotomía de Heller como la prị̂mer línea de tratamiento para la acalasia en la mayoría de los pacientes. Hasta la fecha, sollo cuatro series han presentado datos sustanciale s sobre la evaluación de la ERGE en sus pacientes utilizando los tres métodos (evaluación sistệmática de los síntomas, evaluación endoscópica y estudio ambulatorio de $\mathrm{pH})^{18,19}$. Estos estựdios hallaron que el $27-59 \%$ de los pacientes tenía signos endoscópicos de reflujo (principắ mente esofagitis leve clase A o B de Lòs

TABLA 1. Tasas de éxito en estudios occidentales con el uso de POEM

\begin{tabular}{|c|c|c|c|c|c|c|}
\hline Estudio & Año & $\mathbf{N}$ & $\begin{array}{c}\text { Seguimiento } \\
\text { (meses: mediana) }\end{array}$ & $\begin{array}{l}\text { Puntuación de } \\
\text { Eckardt (pre/ } \\
\text { post) }\end{array}$ & $\begin{array}{c}\text { Presión } \\
\text { EEI (mm Hg) }\end{array}$ & Eficacia (\%) \\
\hline von Renteln D, et al. (13) & 2013 & 70 & 12 & $6.9 / 1$ & $27.6 / 8.9$ & 82.4 \\
\hline Teitelbaum EN, et al. & 2014 & 41 & 12 & $7 / 1$ & $22 / 9$ & 93 \\
\hline Sharata AM, et al. (22) & 2015 & 100 & 21.5 & $6 / 1$ & $44.3 / 19.6$ & 92 \\
\hline Patel KS, et al. (15) & 2015 & 93 & 22 & $7.8 / 6.44$ & $43 / 18$ & 96 \\
\hline Familiari P, et al. (16) & 2016 & 100 & 11 & $8.1 / 1.1$ & $41.4 / 19$ & 94.5 \\
\hline Nieponice A, Nachman F. & 2016 & 50 & 10 & $9 / 1.2$ & $\mathrm{~N} / \mathrm{A}$ & 94.2 \\
\hline
\end{tabular}

EEl: esfínter esofágico inferior. 
Ángeles), el 29-38\% tenía anormalmente elevada la exposición al ácido en los estudios de $\mathrm{pH}$ y el $15-23 \%$ tenían los síntomas frecuentes de reflujo gastroesofágico ${ }^{20,21}$. Estos pacientes han sido tratados eficazmente con IBP. Cabe señalar que las fundoplicaturas de Dor o Toupet realizadas en conjunción con una miotomía de Heller laparoscópica en pacientes con acalasia tienen una eficacia modesta. Estudios de alta calidad de centros expertos en laparoscopia han demostrado que el $18-42 \%$ de los pacientes presentan exposición al ácido anormal en el posoperatorio, similar a lo observado tras un POEM $^{20,21}$. No está claro por qué la tasa de la ERGE después del POEM no es sustancialmente mayor que después de una miotomía de Heller combinada con la fundoplicatura. Puede ser debido al mantenimiento indemne de la anatomía hiatal durante el POEM en comparación con una amplia disección del hiato durante una miotomía estándar. Esta disección extensa interrumpe importantes «ligamentos suspensorios» del esófago, en particular la membrana frenoesofágica, que se cree contribuye al mantenimiento del ángulo de His y tiene una importante función antirreflujo separada del esfínter esofágico propiamente dicho.

\section{NUESTRA EXPERIENCIA}

Entre diciembre de 2013 y agosto de 2016 se analizaron 50 casos de forma prospectiva. El seguimiento medio fue de 10 meses (rango: 6-32). La extensión se limitó hasta obtener una válvula Hill tipo II y nunca excedió de $2 \mathrm{~cm}$. Las variables finales incluyeron el resultado clínico medido por la puntuación ES, la presencia de reflujo sintomático en el Cuestionario de calidad de vida específico para la ERGE, la necesidad de IBP y la esofagitis descartada por endoscopia. El POEM se completó en el $100 \%$ de los pacientes. El seguimiento fue completo en el $100 \%$ de los casos. La eficacia (ES $\leq 3$ ) fue de $47 / 50(94.2 \%$ ) en un seguimiento a corto plazo (seis meses) y $44 / 50$ (88.6\%) en un seguimiento a largo plazo (dos años), con una caída promedio de la ES a partir de 9 a $1.2(\mathrm{p}=0.0001)$. Hubo compli caciones intraoperatorias en dos casos: un sangrado y una perforación mucosa, y en è̀ posoperatorio inmediato un caso de capnotórax masivo, tratadas en forma conservadoră que no requirieron conversión o reinterveñ ción. El promedio de duración de la estancîa hospitalaria fue de 1,3 días. Los casos de rë̀flujo sintomático fueron 10 de $50(20 \%)$, con signos de esofagitis en endoscopia en 4 de $50(8 \%)$. Así mismo, 4 (8\%) requieron contî̀nuar el uso de IBP. En 10/50 casos (20\%) se realizó tratamiento adicional (dilatación endoscópica, promedio 1).

\section{CONCLUSIÓN}

El POEM es un método seguro y eficaz que permite pensar en un cambio de paradigma respecto a la miotomía laparoscópica. Sừ buenos resultados y su baja morbilidad per̂̀miten una recuperación mas rápida dệ paciente, lo cual apoya su adopción. La necè sidad de contar con un equipo multidiscipli nario con amplia experiencia en endoscopie terapéutica hace que sea aconsejable limităr este procedimiento a centros de referencia con alto volumen en esta patología.

\section{BIBLIOGRAFÍA}

\footnotetext{
1. Mayberry JF. Epidemiology and demographics of achalasia. Gastrointest Endosc Clin N Am. 2001;11(2): 235-48.
} 
2. Inoue $\mathrm{H}$, Minami $\mathrm{H}$, Kobayashi $\mathrm{Y}$, et al. Peroral endoscopic myotomy (POEM) for esophageal achalasia. Endoscopy. 2010;42(4):265-71.

3. Pasricha PJ, Hawari R., Ahmed I, et al. Submucosal endoscopic esophageal myotomy: a novel experimental approach for the treatment of achalasia. Endoscopy. 2007;39(9):761-64.

4. Stavropoulos SN, Desilets DJ, Fuchs KH, et al. Per-oral endoscopic myotomy white paper summary. Surg Endosc. 2014 Jul;28(7):2005-19.

5. Pohl D, Tutuian R. Achalasia: an overview of diagnosis and treatment. Gastrointestin Liver Dis. 2007;16(3):297-303.

6. Stavropoulos SN, Modayil R, Friedel D, et al. The international per oral endoscopic myotomy survey (IPOEMS): a snapshot of the global POEM experience. Surg Endosc. 2013;27(9):3322-38.

7. Swanstrom LL, Rieder E, Dunst CM. A stepwise approach and early clinical experience in peroral endoscopic myotomy for the treatment of achalasia and esophageal motility disorders. J Am Coll Surg. 2011;213(6): 751-56.

8. Stefanidis D, Richardson W, Farrell TM, et al. SAGES guidelines for the surgical treatment of esophageal achalasia. Surg Endosc. 2012;26(2): 296-311.

9. Li Q, Chen WF, Zhou PH, et al. Peroral endoscopic myotomy for the treatment of achalasia: a clinical comparative study of endoscopic fullthickness and circular muscle myotomy. J Am Coll Surg. 2014;217(3):420-51.

10. Stavropoulos SN, Desilets DJ, Fuchs KH, et al; NOSCAR POEM White Paper Committee. Per-oral endoscopic myotomy white paper summary. Gastrointest Endosc. 2014;80(1):1-15.

11. Charton JP, Schumacher B, Toermer T, et al Per oral endoscopic myotomy (POEM) for achalasia: pilot case series of a Western referral center. Gastrointest Endosc. 2013;77(5S):AB351.

12. Eckardt AJ, Eckardt V. Treatment and surveillance strategies in achalasia: an update. Nat Rev Gastroenterol Hepatol. 2011;8(6):311-19.
13. Von Renteln D, Fuchs K, Fockens P, et al. Peroral endoscopic myotomy for the treatment of achalasia: an international prospective multicenter study. Gastroenterology. 2013;145:309-11.

14. Kurian AA, Dunst C, Sharata A, et al. Peroral endoscopic esophageal myotomy: defining the learning curve. Gastrointest Endosc. 2013;77(5):719-25

15. Patel KS, Calixte R, Modayil RJ, et al. The light at the end of the tunnel: a single-operator learning curve analysis for per oral endoscopic myotomy. Gastrointest Endosc. 2015;81(5):1181-7.

16. Familiari P, Gigante G, Marchese M, et al. Peroral endoscopic myotomy for esophageal achalasia: outcomes of the first 100 patients with short-term follow-up. Ann Surg. 2016;263(1):82-7.

17. Inoue H, Sato H, Ikeda H, et al. Per-oral endoscopic myotomy: a series 500 patients. J Am Coll Surg. 2015;221(2):256-64.

18. Stavropoulos SN, Modayil RJ, Brathwaite CE, et al. Outcomes of a 5-year, large prospective series of per oral endoscopic myotomy (POEM). Emphasis on objective assessment for GERD and luminal patency. Gastrointest Endosc. 2015;81(5S):AB118-9.

19. Familiari P, Greco S, Gigante G, et al. Gastroesophageal reflux disease aftere peroral endoscopic myotomy: Analysis of clinical, procedural and functional factors, associated with gastroesophageal reflux disease and esophaği tis. Dig Endosc. 2016;28(1):33-41.

20. Kumagai K, Kjellin A, Tsai JA, et al. Toupet versus Dor as a procedureło prevent reflux after cardiomyotomy for achalasia: results of a randomised clinical trial. Int J Surg. 2014;12(7):673-80.

21. Rawlings A, Soper NJ, Oelschlager B, et al. Laparoscopic Dor versus Toupeet fundoplication following Heller myotomy for achalasia: results of a multicenter, prospective, randomized-controlled trial. Surg Endosc. 2012;26(1):18-2̌ 6 .

22. Sharata AM, Dunst CM, Pescarus R, et al. Peroral endoscopic myotomy (POEM) for esophageal primary motility disorders: analysis of 100 consecutive patients. J Gastrointest Surg. 2015;19(1):161-70. 